REVISTA CIENCIAS BIOMÉDICAS

ÉTICA, BIOÉTICA Y HUMANISMO

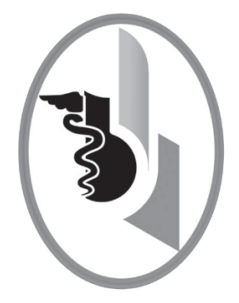

\title{
GESTIÓN DE LA CALIDAD DEL CUIDADO DE ENFERMERÍA CON PRINCIPIOS ÉTICOS*
}

\author{
MANAGEMENT OF NURSING CARE QUALITY WITH ETHICAL \\ PRINCIPLES
}

\author{
Cabarcas Ortega Nirva del Socorro ${ }^{1}$ \\ Correspondencia: E-mail: nicaorte@yahoo.es \\ Recibido para evaluación: septiembre - 12 - 2010 -Aceptado para publicación: enero - 13-2011
}

\section{RESUMEN}

La calidad es un principio rector del cuidado de enfermería, la ley 266 define la calidad como una ayuda eficiente, en términos de oportunidad y uso adecuado de los recursos. La atención de enfermería se basa en el cuidado integral del individuo teniendo como base la dignidad, necesidades derechos y no simplemente la enfermedad. Los cambios y tendencias del cuidado han impuesto nuevos roles en la práctica profesional de la enfermera, presentándose con frecuencia dilemas y conflictos éticos que le exigen tener conocimientos de principios y valores para un mejor análisis de las situaciones y orientación adecuada del ejercicio, debiendo la enfermera hacer uso de sus conocimientos en ética y bioética para la satisfacción de las necesidades del paciente en búsqueda de su bienestar. La gestión de la calidad del cuidado de enfermería implica la planificación, organización, dirección y control de los recursos financieros, humanos y materiales con la intención de cumplir eficazmente con la política de calidad de la institución, teniendo presente los principios éticos establecidos en la ley 266 de 1996. En conclusión la calidad es el resultado de todo el proceso de atención en salud, para llegar a la consecución de esta es necesario implementar un sistema de gestión de la calidad que propenda por lograr el equilibrio entre las necesidades del usuario, infraestructura disponible para la atención, objetivos de la institución, satisfacción de quien provee el cuidado y del usuario. Rev.cienc.biomed. 2011; 2(1): 144-148

\section{PALABRAS CLAVES}

Gestión. Calidad. Cuidado de enfermería. Principios éticos.

\section{SUMMARY}

Quality is a guiding principle of nursing care, law 266 defines it as an efficient help in terms of opportunity and proper use of resources. Nursing care is based on comprehensive care on the basis of individual dignity, rights and needs, not just the disease. Changes and trends in care have imposed new roles in professional practice of nursing, often presenting ethical dilemmas and conflicts that require you to have knowledge of principles and values to better analyze situations and exercise proper orientation; the nurse must use their knowledge in ethics and bioethics to meet the needs of patients for their welfare.

\footnotetext{
* Un producto del Grupo de Investigación Salud Ocupacional perteneciente a la Facultad de Enfermería de la Universidad de Cartagena.

1 Enfermera. Magíster en Enfermería. Docente. Facultad de Enfermería. Universidad de Cartagena. Sub-gerente Científico Servicio de Enfermería y Consulta Externa. Hospital Universitario del Caribe. Cartagena. Colombia.
} 
The management of nursing care quality involves planning, organization, direction and control of financial, human and material resources with the intent to effectively implement the quality policy of the institution, bearing in mind the ethical principles established by law 266 of 1996. In conclusion, the quality is the result of the entire health care process; to reach it, it is necessary to implement a system of quality management that tends to achieve a balance between user needs, available infrastructure for care, objectives of the institution and satisfaction of customer and care providers. Rev. cienc.biomed. 2011; 2(1): 144-148

\section{KEY WORDS}

Management nursing care quality, Ethical principles.

\section{INTRODUCCIÓN}

El ejercicio profesional de enfermería involucra los conceptos de ética, moral, principios éticos, valores éticos en el arte de cuidar, esencia del quehacer de esta profesión, teniéndose como responsabilidades fundamentales el fomentar y restablecer la salud, prevenir la enfermedad, aliviar el sufrimiento, orientando y fundamentados estos cuidados en el respeto a la vida, la dignidad y los derechos humanos.

El cuidar es para enfermería la prestación de un servicio, para lo cual tiene que incorporar prácticas gerenciales, ya que solo a través de la gestión gerencial del cuidado podrá contribuir a su propósito: el cuidado de la salud y la enfermedad del individuo, la familia y la comunidad. El objetivo de la gerencia es la producción de bienes o servicios en las organizaciones. El actuar de la enfermera debe estar fundamentado en principios éticos, normas morales y alimentados por ese cumulo de valores personales y de la profesión que le permitan la toma de decisiones acertada en beneficio de quien recibe los servicios de enfermería, que se espera sean de optima calidad en procura de la satisfacción del usuario del servicio.

La calidad es un concepto integral, en el que se involucran diferente actores: el estado, las empresas promotoras de salud, los prestadores del servicio y los usuarios, debiendo asumir cada uno funciones para propender por la calidad de la atención en salud. Existe una estrecha relación entre la consecución de la calidad y la gestión de esta, es imposible pensar en calidad sin la existencia de la gestión de la calidad con fundamentación ética.

\section{LA CALIDAD EN LO GENERAL}

La búsqueda de la calidad ha llevado a la formulación de múltiples definiciones relacionadas con las actividades propias de la empresa y según quien la define. Esto también ha contribuido al establecimiento de normas de calidad.

Es así como la norma ISO 9000 de uso en todo el mundo desde hace veinte años, define la calidad como "el grado en que un conjunto de características inherentes (rasgos diferenciados) que cumple con los requisitos, generalmente implícitos u obligatorios (habitual a una práctica común para el cliente, la organización y otras partes necesitadas).Contiene especificaciones de tipo técnico administrativo, con el objeto que los productos derivados de los procesos industriales, tengan la posibilidad de ser semejantes en todo el mundo y guarden un índice de calidad semejante.

Para la aplicabilidad de esta norma se debe tener presente que la salud como resultado del proceso de atención no es un producto tangible, por tanto al utilizarse debe hacerse con criterios de flexibilidad, de hecho esta norma está siendo aplicada en el sector salud con éxito.

La evolución en el país del concepto de calidad ha permitido su definición en el marco legal colombiano conferido por la ley 100/93 que en su art. 153, literal 9 dice que "hay calidad, cuando los atributos contenidos en dicha prestación, hacen que la misma sea oportuna, personalizada, integral, continua y de acuerdo con los estándares aceptados en procedimientos y práctica profesional". 
La aplicación del término calidad en salud implica tener presente a que se refiere la calidad de la atención de salud. Definida en el decreto 1011 de 2006 como "la provisión de servicios de salud a los usuarios individuales y colectivos de manera accesible y equitativa, a través de un nivel profesional óptimo, teniendo en cuenta el balance entre beneficios, riesgos y costos, con el propósito de lograr la adhesión y satisfacción de dichos usuarios". Esto hace referencia a la capacidad resolutiva que debe tener la institución que brinda la atención y satisface las necesidades de salud al usuario. Estableciéndose la atención a brindar de acuerdo a los servicios declarados por la institución de salud y al cumplimiento de los estándares de calidad adoptados mediante las resoluciones 1043 y 1445 del 2006, decreto 1011/2006 para el cumplimiento de los requisitos de habilitación y acreditación que deben cumplir los prestadores de servicios de salud.

\section{CALIDAD Y ENFERMERÍA}

En Colombia la calidad está incluida como un principio rector del cuidado de enfermería a través de la ley 266 de 1996 la cual reglamenta su ejercicio profesional. La define como una ayuda eficiente, en términos de oportunidad y uso adecuado de los recursos y además, efectiva; es decir que produzca el impacto deseado para los sujetos receptores del cuidado. Este debe fundamentarse en los valores y estándares técnicos, científicos, sociales, humanos y éticos.

Definida la calidad del cuidado de enfermería como: "La provisión de cuidados básicos, técnicos y de comunicación con oportunidad, pertinencia y continuidad satisfaciendo las necesidades del paciente a fin de mantener o mejorar la calidad de vida del mismo." Vemos como la legislación de calidad de la atención en salud en general en el país está acorde con las exigencias de la calidad de la atención que la enfermera debe brindar al individuo, familia, comunidad en los diferentes niveles de atención, determinándose dicha calidad a través del cumplimiento de las normas preestablecidas; haciéndose necesario el diseño e implementación de estándares de calidad que determinen específicamente la calidad del cuidado de enfermería brindado.
El acto de cuidado de enfermería es el ser y la esencia del ejercicio de la profesión. Se fundamenta en sus propias teorías, tecnologías y conocimientos actualizados de ciencias biológicas, sociales y humanísticas. Se da a partir de la comunicación y relación interpersonal humanizada entre el personal de enfermería y el ser humano, sujeto del cuidado, la familia o grupo social, en las distintas etapas de la vida, situación de salud y del entorno. Implica un juicio de valor y un proceso dinámico y participativo para identificar y dar prioridad a las necesidades y decidir el plan de cuidados de enfermería, con el propósito de promover la vida, prevenir la enfermedad, intervenir en el tratamiento, en la rehabilitación y dar cuidado paliativo con el fin de desarrollar, en lo posible las potencialidades individuales y colectivas.

Todo lo anterior muestra como la calidad es un concepto multidimensional que implica el comportamiento interrelacionado de diversos atributos 0 condicionantes (pertinencia, accesibilidad, continuidad, relaciones interpersonales, comodidad, satisfacción del usuario, oportunidad) en busca de una eficacia y eficiencia de los servicios prestados, haciéndose esto evidente a través de la óptima atención en salud brindada, la cual se refiere a las diferentes acciones y actividades necesarias para promover, mantener y restablecer de manera integral la salud.

De tal manera que brindar atención en salud de calidad implica el cumplimiento satisfactorio e interrelacionado de los atributos de la calidad que se pueden medir a través indicadores de estructura, proceso y productos o resultados. En este quehacer de proferir cuidados de calidad juega un papel importante la institución, la persona que brinda el servicio y el usuario.

\section{LA GESTIÓN DE LA CALIDAD}

La gestión es un proceso que implica el desarrollo de una serie de acciones administrativas para apoyar el desarrollo de los objetivos de la organización. La consecución de la calidad de la atención de la salud incluida en esta la de enfermería, obliga a su gestión, mediante la aplicación de una política de calidad a través de la planificación, organización, control y mejora. 
Para el caso de enfermería, la gestión de la calidad debe ser aplicada mediante la planificación, organización, dirección y control de los recursos financieros, humanos y materiales con la intención de cumplir eficazmente con la política de calidad de la institución. Se debe tener presente que la obtención de la calidad deseada requiere del compromiso y participación de todos, en tanto que la gestión corresponde a la dirección de enfermería.

La gestión en enfermería implica asumir el papel de líder asertivo teniendo en cuenta la satisfacción del cliente interno, externo y el cumplimiento de los objetivos de la institución y a las normas legales existentes. De esta manera la enfermera se enfrenta a grandes retos para la práctica y la gestión del cuidado de enfermería como son el déficit financiero, de enfermeras, de insumos para la atención en salud, las condiciones de práctica, normatividad excesiva, la legislación, las exigencias del usuario cada vez más informado así como los cambios epidemiológicos y demográficos debiendo buscar alternativas que lleven a garantizar la calidad del cuidado al paciente. Es así como la gestión debe estar orientada hacia la persona, el cliente, su familia el personal de enfermería y los equipos interdisciplinarios.

Propender por la calidad en la atención de enfermería no está circunscrito a un nivel ni área de atención en salud específica, esta debe proveerse y gestionarse en todo lugar donde se brinde la atención o el cuidado. Aun así es importante tener presente que algunos niveles (segundo y tercer nivel) y áreas de atención (Hospitalización) se les ha dado mayor importancia en la implementación y evaluación de la calidad de la atención brindada e incluso en el adelanto de investigaciones al respecto, haciéndose necesario ampliar estas acciones al primer nivel y al área ambulatoria e implementar investigaciones sobre el cuidado y la gestión del cuidado con el fin de proponer mejoras en la prestación de los cuidados de enfermería basadas en evidencias científicas.

El profesional de enfermería deberá garantizar cuidados de calidad a quienes reciben sus servicios. Tal garantía no debe entenderse en relación con los resultados de la intervención profesional, dado que el ejercicio de la enfermería implica una obligación de medios, mas no de resultados. La valoración ética del cuidado de enfermería deberá tener en cuenta las circunstancias de tiempo, modo y lugar que rodearon los hechos y las precauciones que frente al mismo hubiera realizado un profesional de enfermería prudente y diligente, de tal manera que la calidad de la atención con principios éticos implica por una parte, de la disposición de adecuadas condiciones de estructura y procesos que disminuyan los posibles riesgos generados de la atención y permitan cumplir con los estándares de calidad y la oportunidad del cuidado y por otro lado es de obligatorio cumplimiento los principios éticos que se encuentran en la Ley 266 de 1996, Capítulo I, Artículo 2, los principios éticos de beneficencia, no maleficencia, autonomía, justicia, veracidad, solidaridad, lealtad y fidelidad, que orientan la responsabilidad deontológica - profesional de enfermería en Colombia.

\section{LA ÉTICA Y LA ENFERMERÍA}

Existen diferentes corrientes que han orientado la ética del cuidado en enfermería contemporánea, entre las cuales encontramos las teorías de Kohlberg, Guilligan, Nooddings. Para Carol Guilligán la toma de decisiones éticas se apoya en la naturaleza del cuidado, haciendo hincapié en el carácter moral. Nel Nooddings combina el conocimiento de la ética con la perspectiva sobre el desarrollo moral de la mujer, centrándose en el valor de los cuidados; el cuidado ético involucra una relación de contacto moral entre dos personas motivadas por el ideal de la solicitud, esta ética del cuidado depende de las condiciones que permitan el florecimiento de la solicitud. La evolución de la ética lleva a tener presente en el actuar profesional las reflexiones hechas por el Dr. Diego Gracia sobre los momentos y horizontes de la ética, contemplándose tres momentos: el primer momento se refiere a la ética del mandato y la obediencia, "origen de la ética", un segundo momento "la ética de derechos y deberes" y un tercer momento "hacia dónde vamos", ética de la obligación, compromiso y responsabilidad, siendo la naturaleza y la 
vida las naturalezas de hombre, el cual debe actuar con autonomía y responsabilidad con sus obligaciones.

Es así como la gestión de la calidad en atención obliga en primera instancia a la realización del diagnóstico institucional y de la demanda en aras de formular una estrategia adecuada que permita planificar, organizar, dirigir y controlar para la implementación del sistema de calidad previamente planificado, basándose en conocimientos técnico científicos, valores y principios éticos, fundamentados en la ética de la responsabilidad.

\section{CONCLUSIONES}

La calidad es el resultado de la buena implementación del proceso de atención en salud con fundamentación científica y ética. Para llegar a la consecución de esta se hace necesario implementar un sistema de gestión de la calidad que propenda por lograr el equilibrio entre las necesidades del usuario, infraestructura disponible para la atención, objetivos de la institución, satisfacción de quien provee el cuidado y del usuario mismo, medido a través de la preservación, mantenimiento y restablecimiento de la salud del cliente con eficiencia y eficacia.

CONFLICTOS DE INTERÉS: ninguno que declarar.

\section{LECTURAS RECOMENDADAS}

1. Abaunza de Gonzalez, M. La investigación y el cuidado en América Latina. Bogotá: Unibiblos; 2005.

2. Albrecht, K. y Zenke, R. Gerencia del servicio. Colombia: Legis Editores; 1991.

3. Alzat E., M. L. Gerencia del cuidado de enfermería, Colombia: Unibiblos; 1997.

4. Bulger, R. J. Creating Nursing's Future, Sullivan, St. Louis Missouri : Ed. Mosby; 1999.

5. Gracia D. Bioética clínica, Santa fé de Bogotá: Editorial el Buho; 1998.

6. Grahan Rumbold, Etica en enfermería, 3a ed, Editorial Mc Graw-Hill Interamericana; 2000.

7. Gracia D. Fundamentación y enseñanza de la bioética. Santa fé de Bogotá: Editorial el Búho; 2004.

8. Peña, B. La investigación y el cuidado en América Latina. Bogotá: Unibiblos; 2005.

9. Universidad de Antioquia. Investigación y educación en enfermería, Vol. XIX No. 2, Colombia: 2001.

10. República de Colombia, Ministerio de la Protección Social, Tribunal Nacional Ético de Enfermería, Ley 911 Código Deontológico de enfermería, Santa féde Bogotá: 2004.

11. Riaño Gamboa Germán, Auditoria de calidad en salud. Santa fé de Bogotá: Grupo Ecomedios; 2001.

12. República de Colombia. Ley 100, por la cual se establece el Sistema General de Seguridad Social en Salud en Colombia, Santa fé de Bogotá: El Ministerio; 1993.

13. República de Colombia. Ministerio de Salud, Decreto 1011 de 2006, por el cual se establece el Sistema Obligatorio de Garantía de Calidad de la Atención de Salud del Sistema General de Seguridad Social en Salud, Santa fé de Bogotá: El Ministerio; 2002.

14. República de Colombia. Ministerio de Salud. Resolución 1043 Por la cual se establecen "las condiciones que deben cumplir los prestadores de servicios de salud para habilitar sus servicios e implementar el componente de auditoría para el mejoramiento de la calidad de la atención y se dictan otras disposiciones", Santa fé de Bogotá: El Ministerio; 2006.

15. República de Colombia. Ministerio de Salud. Resolución 1445. "Define funciones de la entidad acreditadora y se adoptan los manuales de estándares del sistema único de acreditación," Santa fé de Bogotá: 2006. 